\title{
Assessment of Second-Line Antiretroviral Regimens for HIV Therapy in Africa
}

\author{
Nicholas I. Paton, M.D., Cissy Kityo, M.Sc., Anne Hoppe, Ph.D., \\ Andrew Reid, M.R.C.P., Andrew Kambugu, M.Med., Abbas Lugemwa, M.D., \\ Joep J. van Oosterhout, Ph.D., Mary Kiconco, M.P.H., Abraham Siika, M.Med., \\ Raymond Mwebaze, M.Med., Mary Abwola, M.Med., George Abongomera, M.Sc., \\ Aggrey Mweemba, M.Med., Hillary Alima, M.P.H., Dickens Atwongyeire, M.B., Ch.B., \\ Rose Nyirenda, M.Sc., Justine Boles, M.Sc., Jennifer Thompson, M.Sc., \\ Dinah Tumukunde, M.P.H., Ennie Chidziva, Dipl.G.N., Ivan Mambule, M.B., Ch.B., \\ Jose R. Arribas, M.D., Philippa J. Easterbrook, M.D., James Hakim, F.R.C.P., \\ A. Sarah Walker, Ph.D., and Peter Mugyenyi, F.R.C.P., for the EARNEST Trial Team*
}

ABSTRACT

From the Medical Research Council Clinical Trials Unit at University College London, London (N.I.P., A.H., J.B., J.T., A.S.W.); Yong Loo Lin School of Medicine, National University of Singapore, Singapore (N.I.P.); Joint Clinical Research Centre (JCRC) (C.K., D.T., P.M.), Infectious Diseases Institute (A.K., I.M., P.J.E.), and St. Francis of Nsambya Hospital (R.M.), Kampala, JCRC, Mbarara (A.L.), JCRC, Fort Portal (M.K.), JCRC, Mbale (M.A.), JCRC, Gulu (G.A.) JCRC, Kabale (H.A.), and JCRC, Kakira (D.A.) - all in Uganda; University of Zimbabwe Clinical Research Centre, Harare (A.R., E.C., J.H.); Malawi-LiverpoolWellcome Trust Clinical Research Programme, University of Malawi College of Medicine, Blantyre (J.J.O.), Dignitas International, Zomba (J.J.O.), and Mzuzu Central Hospital, Mzuzu (R.N.) - all in Malawi; Moi University School of Medicine, Eldoret, Kenya (A.S.); University Teaching Hospital, Lusaka, Zambia (A.M.); and Hospital La Paz, Madrid (J.R.A.). Address reprint requests to Dr. Paton at the Department of Medicine, Yong Loo Lin School of Medicine, NUHS Tower Block Level 10, $1 E$ Kent Ridge Rd., Singapore 119228, Singapore, or at nick_paton@nuhs.edu.sg.

Drs. Walker and Mugyenyi contributed equally to this article.

*A complete list of members of the EuropeAfrica Research Network for Evaluation of Second-Line Therapy (EARNEST) Trial Team is provided in the Supplementary Appendix, available at NEJM.org.

N EngIJ Med 2014;371:234-47. DOI: 10.1056/NEJMoal311274

Copyright (@) 2014 Massachusetts Medical Society.
BACKGROUND

The efficacy and toxic effects of nucleoside reverse-transcriptase inhibitors (NRTIs) are uncertain when these agents are used with a protease inhibitor in second-line therapy for human immunodeficiency virus (HIV) infection in resource-limited settings. Removing the NRTIs or replacing them with raltegravir may provide a benefit.

METHODS

In this open-label trial in sub-Saharan Africa, we randomly assigned 1277 adults and adolescents with HIV infection and first-line treatment failure to receive a ritonavirboosted protease inhibitor (lopinavir-ritonavir) plus clinician-selected NRTIs (NRTI group, 426 patients), a protease inhibitor plus raltegravir in a superiority comparison (raltegravir group, 433 patients), or protease-inhibitor monotherapy after 12 weeks of induction therapy with raltegravir in a noninferiority comparison (monotherapy group, 418 patients). The primary composite end point, good HIV disease control, was defined as survival with no new World Health Organization stage 4 events, a CD4+ count of more than 250 cells per cubic millimeter, and a viral load of less than 10,000 copies per milliliter or 10,000 copies or more with no protease resistance mutations at week 96 and was analyzed with the use of imputation of data $(\leq 4 \%)$.

RESULTS

Good HIV disease control was achieved in $60 \%$ of the patients (mean, 255 patients) in the NRTI group, $64 \%$ of the patients (mean, 277) in the raltegravir group ( $\mathrm{P}=0.21$ for the comparison with the NRTI group; superiority of raltegravir not shown), and 55\% of the patients (mean, 232) in the monotherapy group (noninferiority of monotherapy not shown, based on a 10-percentage-point margin). There was no significant difference in rates of grade 3 or 4 adverse events among the three groups $(\mathrm{P}=0.82)$. The viral load was less than 400 copies per milliliter in $86 \%$ of patients in the NRTI group, $86 \%$ in the raltegravir group $(\mathrm{P}=0.97)$, and $61 \%$ in the monotherapy group $(\mathrm{P}<0.001)$.

\section{CONCLUSIONS}

When given with a protease inhibitor in second-line therapy, NRTIs retained substantial virologic activity without evidence of increased toxicity, and there was no advantage to replacing them with raltegravir. Virologic control was inferior with protease-inhibitor monotherapy. (Funded by European and Developing Countries Clinical Trials Partnership and others; EARNEST Current Controlled Trials number, ISRCTN 37737787, and ClinicalTrials.gov number, NCT00988039.) 
T

HE PUBLIC HEALTH APPROACH OF THE World Health Organization (WHO), ${ }^{1}$ together with large-scale donor funding, has enabled millions of adults and children in sub-Saharan Africa who are infected with the human immunodeficiency virus (HIV) to have access to lifesaving antiretroviral therapy. ${ }^{2}$ The key principle is the use of simplified, standardized approaches that are feasible on a large scale in resource-limited settings, ${ }^{1,3}$ including a first-line regimen of two nucleoside reverse-transcriptase inhibitors (NRTIs) plus one non-NRTI (NNRTI). In most settings, treatment is monitored clinically and with the use of CD4+ counts, with typically late detection of treatment failure, accompanied by substantial drug resistance. ${ }^{3,4}$ Evidence supporting the WHO-recommended standardized second-line regimen (a boosted protease inhibitor and two NRTIs) is of moderate quality, 3,5 with particular uncertainty around the net contribution of NRTIs. The efficacy of NRTIs is likely to be compromised by cross-resistance from multiple mutations arising during first-line therapy, and the drugs carry well-recognized risks of toxic effects.

We hypothesized that combining a boosted protease inhibitor with raltegravir, a heat-stable integrase inhibitor, to create a second-line regimen with two completely new drug classes that would not be compromised by resistance selected from first-line therapy would sufficiently increase efficacy and decrease toxicity to justify the increased cost of the regimen. Furthermore, we hypothesized that removing NRTIs entirely (using a protease inhibitor alone) would be noninferior to the standard regimen (as has been shown in some first-line studies), ${ }^{6-8}$ with the advantage of reduced toxicity, regimen complexity, and cost.

In the Europe-Africa Research Network for Evaluation of Second-Line Therapy (EARNEST) trial, we evaluated these three options for secondline antiretroviral therapy in an approach that is currently generalizable to sub-Saharan Africa, consisting of clinician-selected NRTIs and clinical and $\mathrm{CD} 4+$ monitoring without resistance testing or regular monitoring of viral load.

\section{METHODS}

\section{STUDY PATIENTS}

From April 12, 2010, to April 29, 2011, we recruited patients at 14 centers in five sub-Saharan African countries. Eligible patients were HIV-infected adults or adolescents ( $>12$ years of age) who had received first-line antiretroviral therapy consisting of two NRTIs and one NNRTI continuously for more than 12 months, had not previously received a protease inhibitor, had missed no more than 3 days of treatment during the preceding month, and had virologic, immunologic, or clinical treatment failure (defined according to WHO 2010 criteria $^{9}$ and confirmed on the basis of a viral load of $>400$ copies per milliliter at screening). Details are provided in the Methods section in the Supplementary Appendix and in the study protocol, both of which are available with the full text of this article at NEJM.org.

Exclusion criteria were current pregnancy or breast-feeding, a life expectancy of less than 1 month, contraindications to any of the study drugs or a requirement for concomitant medications known to interact with any of the study drugs, or known positive results on testing for hepatitis B surface antigen (testing not required at screening).

The protocol was approved by ethics committees in all participating countries and by the research ethics committee at University College London. All patients or their caregivers, in the case of patients less than 18 years of age, provided written informed consent.

\section{STUDY TREATMENT}

Patients were randomly assigned in a 1:1:1 ratio to receive a ritonavir-boosted protease inhibitor, lopinavir-ritonavir (at a dose of $400 \mathrm{mg}$ of lopinavir and $100 \mathrm{mg}$ of ritonavir twice daily), in combination with two or three new or recycled NRTIs chosen without genotyping by the treating clinician (NRTI group); a protease inhibitor in combination with raltegravir (at a dose of $400 \mathrm{mg}$ twice daily) (raltegravir group); or a protease inhibitor alone after raltegravir induction for the first 12 weeks (monotherapy group). The hypotheses were that the raltegravir-containing combination would be superior to the NRTI-containing combination and that protease-inhibitor monotherapy would be noninferior to the NRTI-containing combination.

Randomization was stratified according to study center and CD4+ count $(<200$ cells per cubic millimeter or $\geq 200$ cells per cubic millimeter). The computer-generated, sequentially numbered randomization list with variable block sizes was prepared by the trial statistician and incorporated within the Web-hosted secure database. At each 
center, randomization was performed by the trial manager, who could access the next number but not the whole list.

Clinic visits were scheduled every 4 weeks until week 24, then every 6 weeks until week 48, and then every 8 weeks until week 96 . At each visit, patients were seen by nurses or counselors, with additional assessment by a clinician every other visit; the study drugs were dispensed at each visit. Assessments included symptom review, physical examination, and adherence assessment with the use of standardized questions about missed doses, with further counseling as indicated. A complete blood count was performed and serum alanine aminotransferase and creatinine levels were measured at weeks 12,48 , and 96, and a CD4+ count was performed every 12 weeks until week 48 and then every 16 weeks, with all laboratory testing performed locally and results returned to the treating clinician. The CockcroftGault equation was used to calculate the estimated glomerular filtration rate. Additional testing could be performed at the discretion of the treating clinician.

Although there was no routine local monitoring of viral load, in cases in which there was clinical or immunologic failure (as defined in the Methods section in the Supplementary Appendix) and an alternative regimen was available locally, clinicians could perform local open viralload testing after approval from an expert review committee and could change the treatment if appropriate. Substitutions in the antiretroviral regimen within drug classes were allowed in cases of toxicity. Women in the monotherapy group who became pregnant added NRTIs during the period in which they were either pregnant or breast-feeding. Tuberculosis was treated with the use of rifabutin, with no changes in the study-drug regimens.

\section{LABORATORY ANALYSES}

Viral load was measured centrally at the Joint Clinical Research Centre (JCRC) in Kampala, Uganda, on samples that were stored at week 48 and week 96. Laboratory staff members who were unaware of study-group assignments performed the analyses using the Abbott RealTime HIV-1 assay. Laboratory staff members at Janssen Diagnostics in Beerse, Belgium, performed genotypic resistance testing by sequencing reverse transcriptase, protease, and integrase genes, according to study group, in a blinded fashion on all postrandomization samples with a viral load of more than 1000 copies per milliliter. Genotyping (reverse transcriptase) of baseline samples from patients in the NRTI group was performed at the JCRC in Kampala. Drug-susceptibility predictions were made with the use of the Stanford algorithm..$^{10}$ Results of viral-load measurement and genotyping were not returned to the treating clinicians but were reviewed by an independent data and safety monitoring committee.

\section{EVENT ADJUDICATION}

The expert review committee, made up of four independent physicians with experience in treating patients with HIV infection, adjudicated reported adverse events against the following standard prespecified diagnostic criteria: for stage 3 and 4 events, WHO criteria ${ }^{11}$; for serious HIVassociated events not diagnostic of the acquired immunodeficiency syndrome (AIDS), criteria based on those of the International Network for Strategic Initiatives in Global HIV Trials (INSIGHT) ${ }^{12}$; and for all adverse events, the criteria of the Division of AIDS of the National Institutes of Health. ${ }^{13}$ Committee members also assessed the relationship between such events and the study drugs.

\section{STUDY END POINTS}

The primary composite end point, good HIV disease control, was defined as no new WHO stage 4 events (other than esophageal candidiasis or mucosal herpes simplex virus infection) or death, a CD4+ count of more than 250 cells per cubic millimeter, and a viral load of less than 10,000 copies per milliliter (or $\geq 10,000$ copies per milliliter without major or minor protease-inhibitor resistance mutations) at week 96 . This end point was designed to be pragmatic and to reflect realistic outcomes for a public health approach and overall patient well-being. (Further rationale for the primary end point and a list of secondary end points are provided in the Methods section in the Supplementary Appendix.)

The protocol specified an analysis of the primary end point at 96 weeks, but patients were to be followed for 144 weeks. The data and safety monitoring committee reviewed interim data approximately annually (at five meetings) with the 
use of the Haybittle-Peto method $(\mathrm{P}<0.001) .{ }^{14}$ At their last meeting (on April 15, 2013) after the last week 96 visit, committee members recommended that the monotherapy group be stopped on the basis of the substantially inferior level of viral-load suppression at week 96 .

\section{STUDY OVERSIGHT}

The pharmaceutical industry partners donated medications, test kits, in-kind services, and funding but had no role in the study design, data collection (other than genotyping), data analysis, writing of the manuscript, or the decision to submit the manuscript for publication. All authors vouch for the completeness of the data and analyses presented and the fidelity of this report to the protocol.

\section{STATISTICAL ANALYSIS}

We estimated that the enrollment of 400 patients in each of the three study groups would provide a power of $80 \%$ to show the noninferiority of protease-inhibitor monotherapy, which was defined as an absolute reduction in response of no more than 10 percentage points, as compared with the NRTI group. This estimation was based on the assumption that $75 \%$ of the patients in the NRTI group would have good HIV disease control at week 96 (for details, see the Methods section in the Supplementary Appendix), with a onesided alpha level of 0.025 (because there were two randomized comparisons) and assuming that $10 \%$ of patients would be lost to follow-up. The noninferiority margin of 10 percentage points was based on Food and Drug Administration guidance. ${ }^{15,16} \mathrm{We}$ also estimated that the enrollment of 400 patients in each study group would provide a power of $87 \%$ to show the superiority of the raltegravir regimen if the response was 10 percentage points higher than that in the NRTI group, with a two-sided alpha level of 0.025 and assuming that $10 \%$ of patients would be lost to follow-up.

All comparisons were performed in the intention-to-treat population regardless of changes in antiretroviral therapy after randomization. All the results presented here are based on two-sided statistical tests without adjustment for multiple testing. The $95 \%$ confidence intervals that are presented correspond to a two-sided test for superiority; for comparisons assessing noninferiority, the focus is on the lower confidence interval. Good HIV disease control at week 96 was compared among the study groups with the use of absolute risk differences and logistic regression, with multiple imputation for missing CD4+ counts, viral loads, and genotypes (which accounted for $\leq 4 \%$ of observations) (Fig. 1). (For details, see the Methods section in the Supplementary Appendix. $)^{17,18}$

Complete case analysis was used for all secondary end points, with the exclusion of deaths, loss to follow-up, and missed visits. Binary end points were compared with the use of the risk difference and chi-square tests; continuous variables were compared with the use of the mean change from baseline and t-tests or analysis of variance. Generalized estimating equations (binomial or normal distribution, respectively, both with independent correlation structure) were used as tests of difference between study groups during all visit weeks. We used Cox proportionalhazards regression to analyze time-to-event end points. (See the Methods section in the Supplementary Appendix for details with respect to the statistical analyses.)

\section{RESULTS}

\section{STUDY PATIENTS}

Of the 1277 patients who underwent randomization, 5 did not meet all entry criteria but were included in the analyses, since they all had failure of first-line treatment (Table S1 in the Supplementary Appendix). Failure of first-line treatment was well established, with $42 \%$ of patients having a viral load of more than 100,000 copies per milliliter, $62 \%$ with a CD4+ count of less than 100 cells per cubic millimeter, and extensive NRTI resistance, including $57 \%$ with intermediate- or high-level resistance to tenofovir (Table 1, and Table S2 in the Supplementary Appendix). The NRTIs that were most frequently selected in the NRTI group were tenofovir plus either lamivudine or emtricitabine (in 70\% of patients), with additional zidovudine in another $9 \%$ of patients (in accordance with national guidelines in Malawi) (Table S3 in the Supplementary Appendix).

A total of $6 \%$ of the study patients died before week $96,0.7 \%$ withdrew from the study, and $0.7 \%$ were lost to follow-up, with similar rates in the three study groups (Fig. 1). Among survivors 


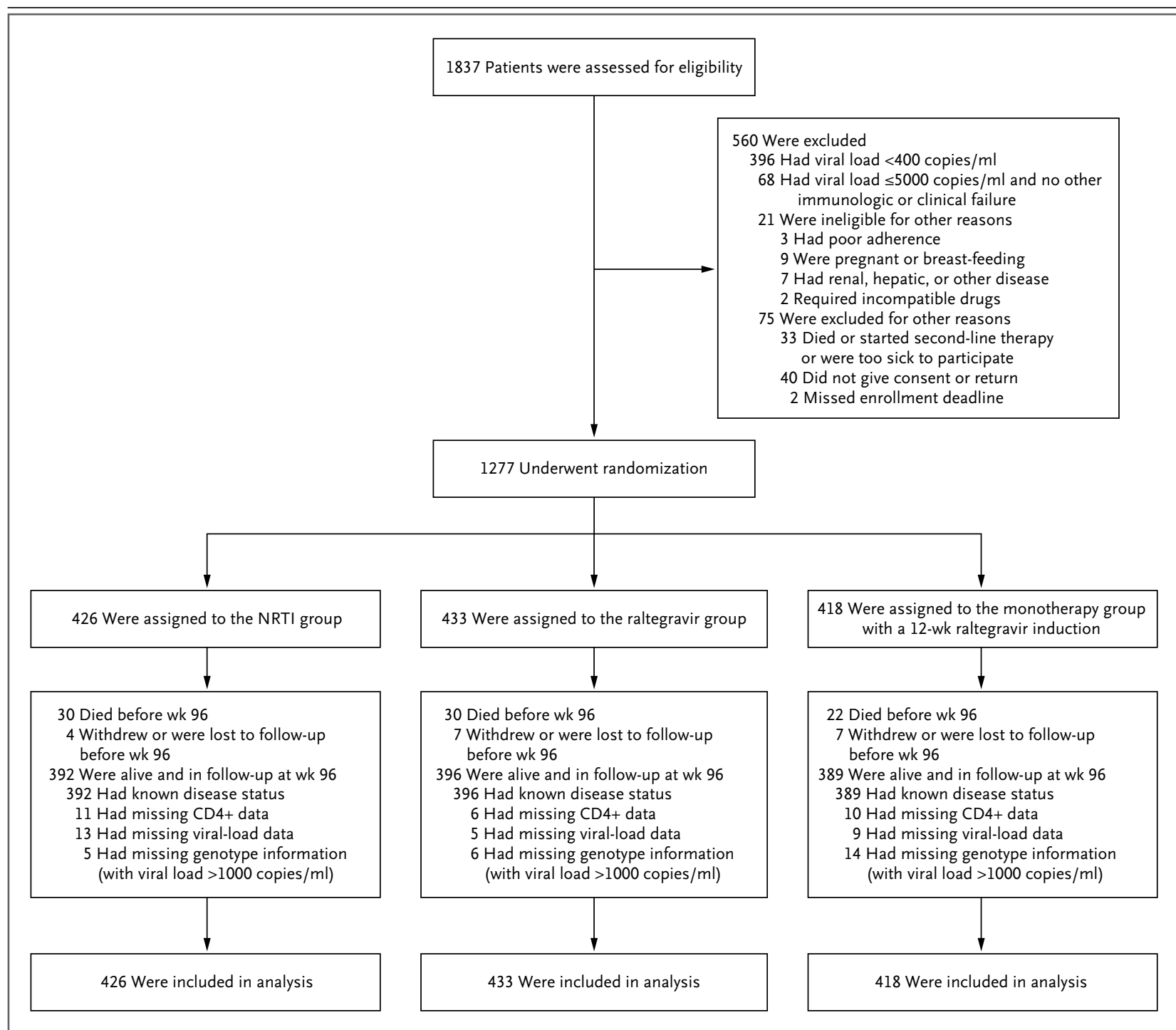

Figure 1. Enrollment and Outcomes.

At week 96, data for patients with missing values for the CD4+ count, viral load, or genotype were imputed for the analysis of the primary end point, according to the prespecified statistical analysis plan.

at week 96, the proportions of patients who were still taking the appropriate classes of drugs for their assigned group were $99 \%$ in the NRTI group, $97 \%$ in the raltegravir group, and $98 \%$ in the monotherapy group $(\mathrm{P}=0.23$ for the comparison among the three groups); overall, the proportions of person-time receiving drugs consistent with the assigned strategy were $99 \%, 97 \%$, and $97 \%$ in the three groups, respectively, with the allowance of within-class substitutions and NRTIs added to the regimen in the monotherapy group in case of pregnancy. Self-reported adherence to study medication was high. The proportions of visits at which patients reported no missed medication doses in the previous month were $87 \%$ in the NRTI group, $89 \%$ in the raltegravir group, and $88 \%$ in the monotherapy group $(\mathrm{P}=0.26$ for the comparison among the three groups); the respective proportions of visits with no missed doses on weekends were 96\%, 97\%, and 96\% ( $\mathrm{P}=0.23$ ), and the proportions with no missed doses for 4 or more consecutive days were $98 \%, 99 \%$, and $98 \%(P=0.30)$. 


\section{EFFICACY END POINTS}

Good HIV disease control (the primary end point) was achieved in $60 \%$ of the patients in the NRTI group (imputed mean based on multiple imputations from 25 simulations, 255 patients), 64\% in the raltegravir group (imputed mean, 277 patients), and 55\% in the monotherapy group (imputed mean, 232 patients). Patients receiving raltegravir, as compared with those receiving NRTIs, had a nonsignificant increase in the primary end point of 4.2 percentage points $(95 \%$ confidence interval [CI], -2.4 to $10.7 ; \mathrm{P}=0.21$; superiority not shown). Patients receiving protease-inhibitor monotherapy, as compared with those receiving NRTIs, had a nonsignificant decrease in the primary end point of -4.4 percentage points ( $95 \%$ CI, -11.2 to 2.4 ; noninferiority not shown, since the lower boundary exceeded the prespecified margin of 10 percentage points) (Table 2). The results did not differ significantly when observed data without imputation were used in the analysis (Table S5 in the Supplementary Appendix).

Similar proportions of patients $(\geq 90 \%)$ in the three study groups were alive at week 96 with no new WHO grade 4 events ( $\mathrm{P}=0.73$ for the comparison among the three groups) (Table 2 , and Table S11 and Fig. S1 in the Supplementary Appendix). At 96 weeks, CD4+ counts were higher than 250 cells per cubic millimeter in 251 of 381 patients (66\%) in the NRTI group, 280 of 390 patients $(72 \%)$ in the raltegravir group, and 243 of 379 patients (64\%) in the monotherapy group $(\mathrm{P}=0.08$ for the comparison between the NRTI group and the raltegravir group). The mean increase in the CD4+ count at week 96 tended to be greater in the raltegravir group than in the NRTI group ( $\mathrm{P}=0.05$ ) (Table 2, and Fig. $\mathrm{S} 2$ in the Supplementary Appendix). Fewer patients in the monotherapy group than in the other two groups met the criterion for good disease control with respect to viral load $(\mathrm{P}<0.001$ for the comparison among the three groups). Sensitivity analyses showed similar results (Tables S5 and S7 in the Supplementary Appendix).

A viral load of less than 400 copies per milliliter at week 96 was reported in $86 \%$ of patients in the NRTI group as compared with $86 \%$ in the raltegravir group (absolute risk difference, -0.1 percentage points; $95 \% \mathrm{CI},-5.0$ to $4.8 ; \mathrm{P}=0.97$ ) and $61 \%$ in the monotherapy group (absolute risk difference, -24.7 percentage points; $95 \% \mathrm{CI},-30.7$ to -18.7 ;
$\mathrm{P}<0.001)$ (Table 2 and Fig. 2A). There was no significant difference between the NRTI group and the raltegravir group at any viral-load threshold that was evaluated ( $P>0.30$ for all comparisons), whereas the proportion of patients with viralload suppression was significantly lower in the monotherapy group than in the NRTI group at all viral-load thresholds $(\mathrm{P}<0.01$ for all comparisons), differences that were progressively larger at lower thresholds (Table 2 and Fig. 2A, and Table S6 in the Supplementary Appendix). No important heterogeneity was identified among groups (Tables S4 and S6 in the Supplementary Appendix).

\section{RESISTANCE MUTATIONS}

Intermediate- or high-level resistance to lopinavir was present at week 96 in $2 \%$ of patients in the NRTI group, $1 \%$ of those in the raltegravir group, and $18 \%$ of those in the monotherapy group ( $\mathrm{P}<0.001$ for the comparison among the three groups) (Table 2 and Fig. 2B). Among the genotype samples obtained from 194 patients, the major protease-inhibitor resistance mutations that were seen most frequently were $82 \mathrm{~A}$ in $47 \mathrm{pa}-$ tients (24\%), $46 \mathrm{I}$ in 43 patients (22\%), and $76 \mathrm{~V}$ in 22 patients (11\%). In the NRTI group, $4 \%$ of patients had intermediate- or high-level resistance to one or more NRTIs (with the exclusion of lamivudine and emtricitabine) taken during the trial; mutations included 70R in 10 patients, $67 \mathrm{~N}$ in 9 patients, and 65R in 1 patient; mutations in TAM2 and TAM1 were found in 36 and 14 patients, respectively. In the raltegravir group, $3 \%$ of patients had intermediate- or high-level resistance mutations to raltegravir (2 patients with 143R, 3 patients with $155 \mathrm{H}$, and 1 patient with $140 \mathrm{~S}+148 \mathrm{H})$. In the monotherapy group, $4 \%$ of patients had intermediate-level resistance mutations and no patients had high-level resistance mutations to darunavir.

\section{ADVERSE EVENTS}

There were 312 serious adverse events (of which 82 were fatal), 414 grade 3 or 4 adverse events (of which 61 were classified by the expert review committee as possibly, probably, or definitely related to a study drug), and 3 serious non-AIDS events, with no significant between-group differences ( $\mathrm{P}>0.18$ for all comparisons) (Table 3 , and Tables S10 and S12 and Fig. S4 and S5 in the Supplementary Appendix). A total of 48 patients 
(4\%) had their drug regimen modified because of an adverse event $(\mathrm{P}=0.28$ for the comparison among the three groups); the most common reasons reported were hematologic events in 4 patients in the NRTI group, rash or hypersensitivity reactions in 9 patients and hepatic events in 6 patients in the raltegravir group, and hepatic events in 6 patients in the monotherapy group. At week 96, reductions in the estimated glomerular filtration rate were greater in the NRTI group $(\mathrm{P}=0.02$ as compared with the raltegravir group and $\mathrm{P}=0.06$ as compared with the monotherapy group), but there was no evidence of significant between-group differences in the proportion of patients with a rate of less than $60 \mathrm{ml}$ per minute per $1.73 \mathrm{~m}^{2}(\mathrm{P}=0.22$ for the comparison among the three groups) or the magnitude of the increase in the hemoglobin level $(\mathrm{P}=0.13$ for the comparison among the three groups) (Table 3 , and Fig. S6 and S7 in the Supplementary Appendix).

\section{SCUSSION}

In a randomized, controlled trial of second-line therapy in low-income countries in sub-Saharan Africa, we found that the WHO-recommended regimen of a boosted protease inhibitor (in this case, lopinavir) combined with two NRTIs was

\begin{tabular}{|c|c|c|c|c|}
\hline Characteristic & $\begin{array}{c}\text { NRTI } \\
\text { Group } \\
(N=426)\end{array}$ & $\begin{array}{l}\text { Raltegravir } \\
\text { Group } \\
(\mathrm{N}=433)\end{array}$ & $\begin{array}{c}\text { Monotherapy } \\
\text { Group } \\
(\mathrm{N}=418)\end{array}$ & $\begin{array}{l}\text { All Patients } \\
(\mathrm{N}=1277)\end{array}$ \\
\hline Female sex 一 no. (\%) & $264(62)$ & $263(61)$ & $215(51)$ & $742(58)$ \\
\hline \multicolumn{5}{|l|}{ Age $-y r$} \\
\hline Median (IQR) & $37(31-43)$ & $37(30-43)$ & $38(32-44)$ & $37(31-44)$ \\
\hline Range & $12-73$ & $12-75$ & $12-71$ & $12-75$ \\
\hline Median weight (IQR) — kg & $56.0(48.9-63.7)$ & $55.8(48.8-64.0)$ & $56.5(50.0-64.8)$ & $56.0(49.2-64.0)$ \\
\hline Median body-mass index (IQR) & $20(18-23)$ & $21(18-23)$ & $21(18-23)$ & $21(18-23)$ \\
\hline \multicolumn{5}{|l|}{ Disease status } \\
\hline \multicolumn{5}{|l|}{ WHO stage - no. (\%) } \\
\hline Patients with available data & $244(57)$ & $264(61)$ & $258(62)$ & $766(60)$ \\
\hline 2 & $51(12)$ & $49(11)$ & $52(12)$ & $152(12)$ \\
\hline 3 & $108(25)$ & $117(27)$ & $109(26)$ & $334(26)$ \\
\hline 4 & $85(20)$ & $98(23)$ & $97(23)$ & $280(22)$ \\
\hline \multicolumn{5}{|l|}{ CD4+ count } \\
\hline Median (IQR) - cells $/ \mathrm{mm}^{3}$ & $72(29-143)$ & $70(27-142)$ & 70 (33-149) & $71(30-146)$ \\
\hline$<100$ cells $/ \mathrm{mm}^{3}-$ no. (\%) & $262(62)$ & $267(62)$ & $258(62)$ & $787(62)$ \\
\hline \multicolumn{5}{|l|}{ Highest CD4+ count during first-line therapy } \\
\hline Patients with available data - no. (\%) & $330(77)$ & $351(81)$ & $338(81)$ & $1019(80)$ \\
\hline Median (IQR) - cells $/ \mathrm{mm}^{3}$ & $257(150-383)$ & $233(141-392)$ & $236(139-363)$ & $243(142-375)$ \\
\hline \multicolumn{5}{|l|}{ Viral load } \\
\hline Median (IQR) - copies/ml & $\begin{array}{c}67,515 \\
(23,065-175,800)\end{array}$ & $\begin{array}{c}74,500 \\
(25,004-205,000)\end{array}$ & $\begin{array}{c}70,874 \\
(21,584-210,000)\end{array}$ & $\begin{array}{c}69,782 \\
(23,183-194,690)\end{array}$ \\
\hline$\geq 100,000$ copies $/ \mathrm{ml}$ - no. (\%) & $168(39)$ & $181(42)$ & $181(43)$ & $530(42)$ \\
\hline \multicolumn{5}{|l|}{ Previous antiretroviral therapy } \\
\hline Median duration (IQR) - yr & $4.0(2.8-5.4)$ & $4.0(2.9-5.5)$ & $3.9(2.6-5.4)$ & $4.0(2.8-5.4)$ \\
\hline \multicolumn{5}{|l|}{ Drug - no. (\%) } \\
\hline Zidovudine & $292(69)$ & $283(65)$ & $287(69)$ & $862(68)$ \\
\hline Stavudine & $266(62)$ & $266(61)$ & $245(59)$ & $777(61)$ \\
\hline Tenofovir & $52(12)$ & 71 (16) & 60 (14) & $183(14)$ \\
\hline
\end{tabular}




\begin{tabular}{|c|c|c|c|c|}
\hline Characteristic & $\begin{array}{c}\text { NRTI } \\
\text { Group } \\
(\mathrm{N}=426)\end{array}$ & $\begin{array}{l}\text { Raltegravir } \\
\text { Group } \\
(\mathrm{N}=433)\end{array}$ & $\begin{array}{c}\text { Monotherapy } \\
\text { Group } \\
(\mathrm{N}=418)\end{array}$ & $\begin{array}{l}\text { All Patients } \\
(\mathrm{N}=1277)\end{array}$ \\
\hline \multicolumn{5}{|l|}{ Treatment-failure criteria fulfilled — no. (\%) } \\
\hline WHO stage 4 & $62(15)$ & $65(15)$ & $69(17)$ & $196(15)$ \\
\hline \multicolumn{5}{|l|}{ CD4+ count } \\
\hline Latest count $<100 / \mathrm{mm}^{3}$ & $262(62)$ & $267(62)$ & $258(62)$ & $787(62)$ \\
\hline $\begin{array}{l}\text { Latest count below count preceding initiation of } \\
\text { first-line therapy }\end{array}$ & $165(39)$ & $154(36)$ & $155(37)$ & $474(37)$ \\
\hline $\begin{array}{l}\text { Latest count }<200 / \mathrm{mm}^{3} \text { with at least one previ- } \\
\text { ous count }>400 / \mathrm{mm}^{3}\end{array}$ & $47(11)$ & $49(11)$ & $40(10)$ & $136(11)$ \\
\hline Latest viral load $>5000$ copies $/ \mathrm{ml}$ & $402(94)$ & $403(93)$ & $388(93)$ & $1193(93)$ \\
\hline \multicolumn{5}{|l|}{ Laboratory values $\rrbracket$} \\
\hline Hemoglobin - g/dl & $11.9 \pm 2.2$ & $11.9 \pm 2.2$ & $12.0 \pm 2.1$ & $11.9 \pm 2.2$ \\
\hline Creatinine $-\mathrm{mg} / \mathrm{dl}$ & $0.74 \pm 0.28$ & $0.76 \pm 0.37$ & $0.78 \pm 0.37$ & $0.76 \pm 0.34$ \\
\hline \multicolumn{5}{|l|}{ Estimated glomerular filtration rate } \\
\hline Mean $-\mathrm{ml} / \mathrm{min} / 1.73 \mathrm{~m}^{2}$ & $114.7 \pm 37.6$ & $114.8 \pm 39.1$ & $112.5 \pm 38.0$ & $114.0 \pm 38.2$ \\
\hline$<60 \mathrm{ml} / \mathrm{min} / 1.73 \mathrm{~m}^{2}-$ no. (\%) & $17(4)$ & $18(4)$ & $25(6)$ & $60(5)$ \\
\hline \multicolumn{5}{|c|}{$\begin{array}{l}\text { Plus-minus values are means } \pm \text { SD. There were no significant differences among the groups except for sex }(P=0.003) \text {. To convert the values } \\
\text { for creatinine to micromoles per liter, multiply by } 88.4 \text {. IQR denotes interquartile range, and WHO World Health Organization. } \\
\text { The body-mass index (the weight in kilograms divided by the square of the height in meters) was calculated for } 1243 \text { of the } 1277 \text { patients } \\
(97 \%) \text {. } \\
\text { WHO stage was based on } 2006 \text { WHO case definitions. }{ }^{11} \\
\text { Values for hemoglobin, creatinine, and the estimated glomerular filtration rate were available for } 1268 \text { patients (99\%), } 1247 \text { patients (98\%), } \\
\text { and } 1238 \text { patients }(97 \%) \text {, respectively. }\end{array}$} \\
\hline
\end{tabular}

effective and had an acceptable safety profile, with a $90 \%$ rate of survival free of WHO stage 4 events and an $86 \%$ rate of virologic suppression ( $<400$ copies per milliliter) at 96 weeks. Although outcomes are often better in clinical trials than in clinical practice, our entry criteria were broadly generalizable, and we followed the widely used approach of clinical and CD4+ monitoring, without viral-load testing (apart from targeted testing to confirm treatment failure before changing therapy). At all study centers, we followed standard practice with respect to adherence counseling, which is a low-technology intervention that can be replicated in resource-limited settings. The good outcomes in the NRTI group in this trial are also consistent with the results of previous cohort studies in several resource-limited settings. ${ }^{19} \mathrm{Al}-$ though CD4+ counts increased, indicating immunologic recovery, only $66 \%$ of patients in the NRTI group had a CD4+ count of more than 250 cells per cubic millimeter by 96 weeks, which probably reflects the low baseline CD4+ counts in patients at the time of the switch to second- line therapy. ${ }^{20}$ However, even with persistently low CD4+ counts, patients receiving antiretroviral therapy would be expected to have reduced rates of HIV-related complications. ${ }^{21}$

Raltegravir was not superior to NRTIs when used in combination with a protease inhibitor in second-line therapy - an unexpected finding, given the predicted benefit associated with adding a second new drug class without overlap with first-line treatment regimens. We considered it important to show that the raltegravir combination was superior, given the substantial additional cost of this agent, as compared with the cost of NRTIs, which is a major disadvantage for national treatment programs with finite resources. Nevertheless, the raltegravir combination was noninferior to the NRTI combination for all primary and secondary end points, findings that are consistent with the results of a trial of lopinavir and raltegravir, as compared with lopinavir and NRTIs, as first-line therapy in high-income countries (with presumably full NRTI activity) ${ }^{22}$ and a trial of second-line therapy in high- and middle- 


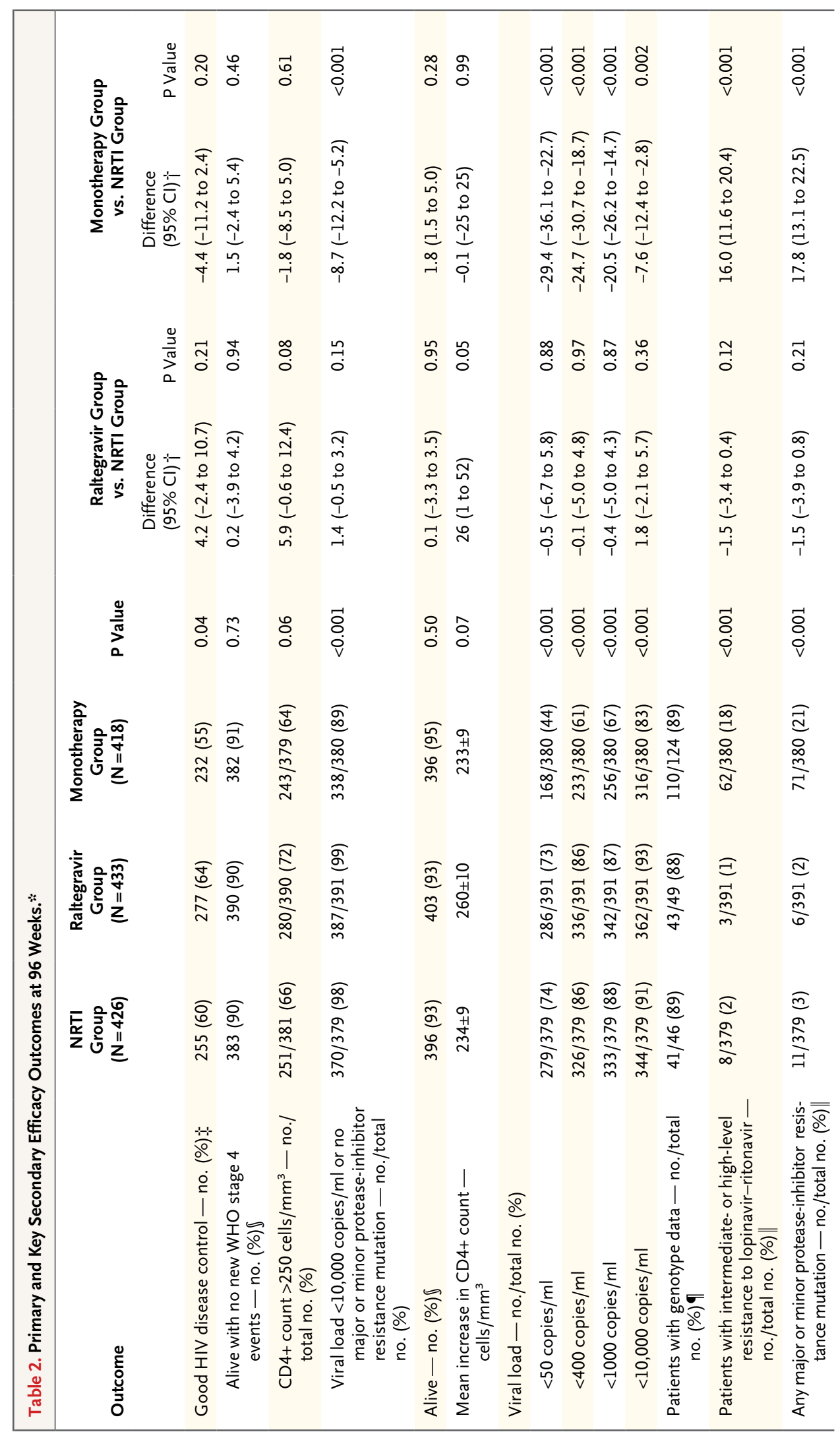




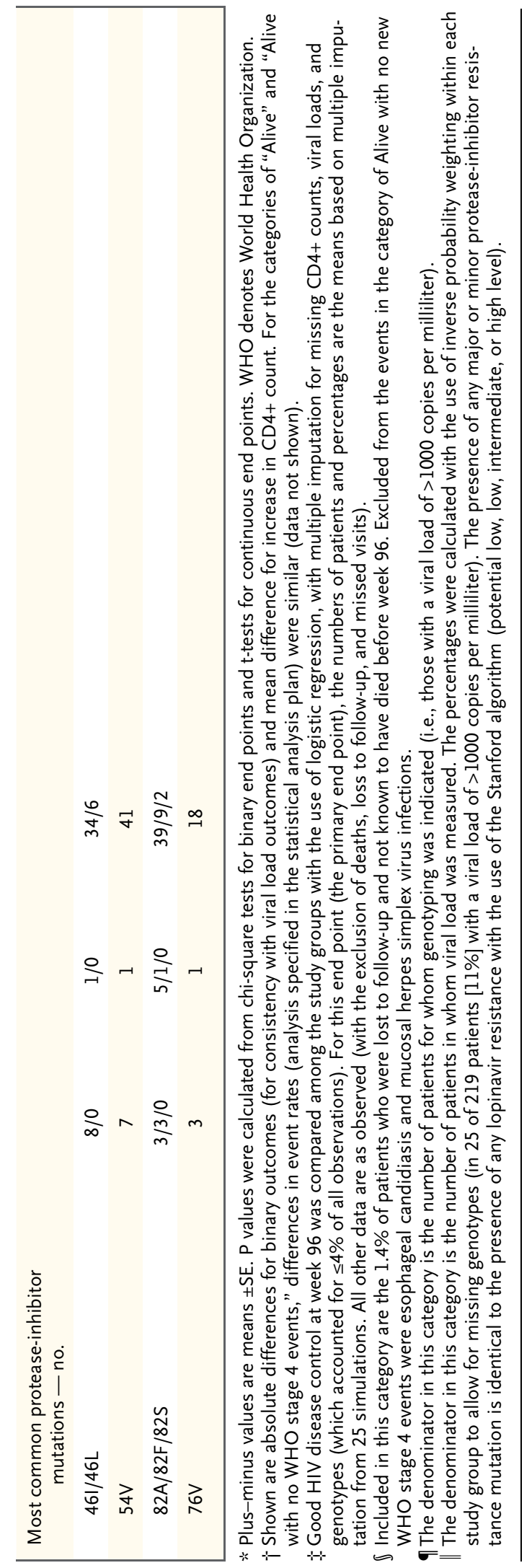

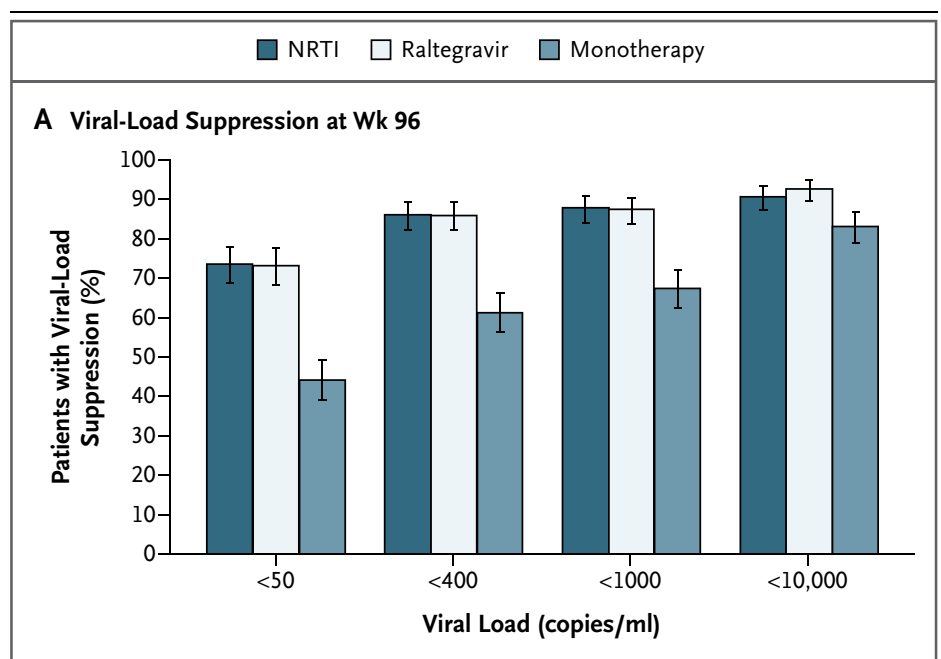

B Drug Resistance at Wk 96

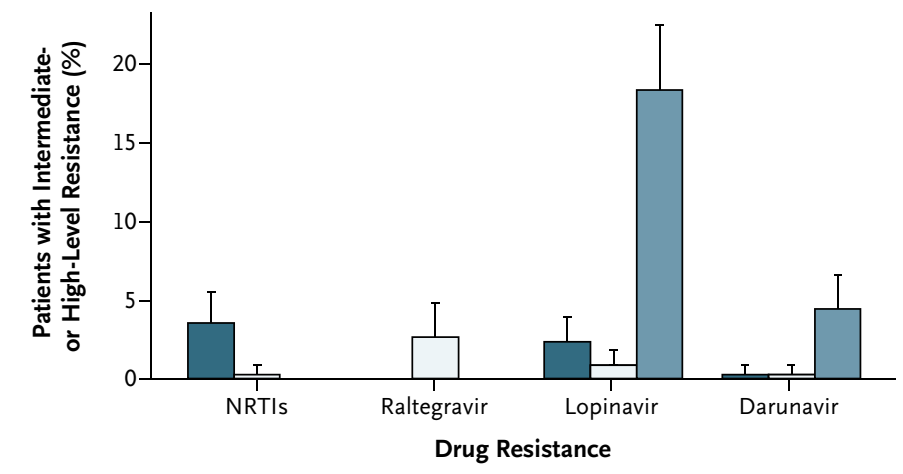

Figure 2. Viral-Load Suppression and Drug Resistance at Week 96.

Panel A shows the proportion of patients with various levels of viral-load suppression. Panel B shows the proportion of patients with intermediate- or high-level drug resistance. Resistance to NRTIs is limited to drugs taken during the trial and excludes resistance to lamivudine or emtricitabine. In the two panels, I and T bars indicate $95 \%$ confidence intervals. The outcomes at week 48 are provided in Figure S3 in the Supplementary Appendix.

income countries with mainly genotyping-guided selection of NRTIs and viral-load monitoring. ${ }^{23}$ We also found that the raltegravir combination had a safety profile similar to that of the NRTI combination and may therefore be an alternative second-line regimen in resource-rich settings where individualized therapy is feasible. The trend toward a modest advantage of raltegravir with respect to the increase in the CD4+ count, without differences in viral-load suppression, may reflect independent effects of raltegravir on T-cell activation or survival, ${ }^{24,25}$ but there was no associated clinical benefit at week 96. Follow-up to week 144 will assess longer-term outcomes and cost-effectiveness, 


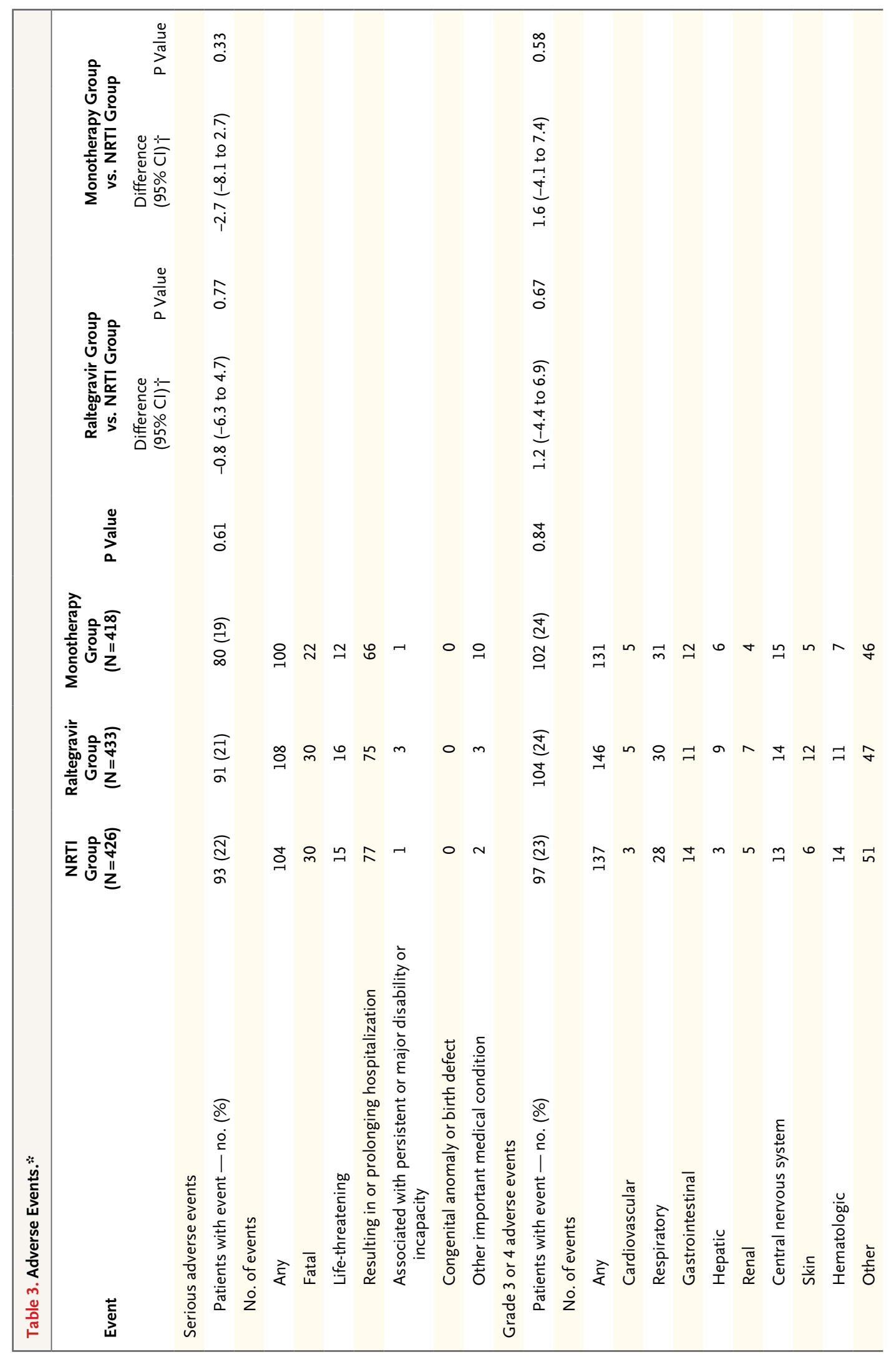




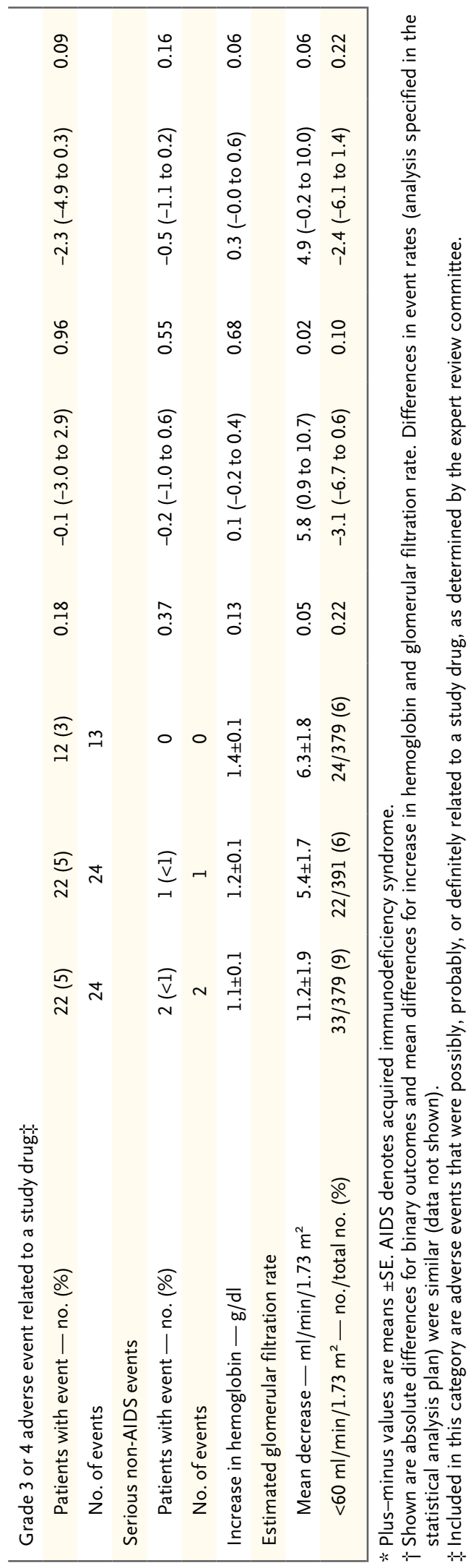

data that are essential for evaluating a new regimen before large-scale implementation.

We did not establish the noninferiority of protease-inhibitor monotherapy to combination therapy with NRTIs with respect to the primary end point, and monotherapy resulted in inferior viral-load suppression, which was associated with increased protease-inhibitor resistance. On the basis of these findings, the data and safety monitoring committee recommended the discontinuation of the monotherapy group, and all patients in that group were switched to combination therapy. Several previous, noncomparative studies and one small, randomized study have suggested that protease-inhibitor monotherapy may be promising for second-line therapy in resource-limited settings. ${ }^{26-28}$ The other small 48-week comparative trial in Thailand showed that protease-inhibitor monotherapy was inferior to protease-inhibitor therapy plus NRTIs on the basis of lower rates of viral-load suppression to a level under 50 copies per milliliter (with only a trend toward lower rates of suppression to a level under 400 copies per milliliter and little protease-inhibitor resistance). ${ }^{29}$

Since outcomes with protease-inhibitor monotherapy are better when treatment is initiated after viral-load suppression, ${ }^{6,30,31}$ we used an induction approach with raltegravir. A longer induction period or a strategy including regular viral-load monitoring with immediate NRTI reintensification after viral-load rebound might have produced better outcomes, but such a regimen would be challenging to implement and would diminish the potential practical and cost advantages of protease-inhibitor monotherapy in resourcelimited settings. Although protease-inhibitor monotherapy may be a reasonable management strategy for individualized therapy, our findings indicate that it is not appropriate as a standardized regimen in the public health approach. Nevertheless, the similar clinical and CD4+ outcomes in the NRTI and monotherapy groups provide reassurance that even when a regimen does not fully suppress the viral load, it can still provide benefits.

The comparison of the NRTI group with the monotherapy group allowed us to assess the contribution of NRTIs to second-line regimens in a population with first-line treatment failure and extensive NRTI cross-resistance, with NRTIs selected on the basis of simple algorithms with- 
out genotypic testing. In this setting, which is typical of most sub-Saharan African antiretroviral programs, the finding of substantial residual NRTI activity was remarkable. This may partly derive from the first use of tenofovir as a secondline therapy in many patients, although a substantial proportion of patients had tenofovir resistance at baseline. Results may also reflect residual antiviral-drug activity despite resistance or maintenance of viral populations with reduced replicative capacity (especially with the use of lamivudine, with the maintenance of the M184V mutation). ${ }^{32,33}$ Our findings can probably be generalized to settings in which viral-load monitoring is used to detect first-line treatment failure earlier with less cross-resistance or settings in which resistance testing is available to guide the selection of NRTIs. In such settings, viral-load suppression in patients receiving combination therapy with an NRTI and a protease inhibitor might improve slightly, but this would decrease the likelihood of finding that a raltegravir combination is superior or that protease-inhibitor monotherapy is noninferior to an NRTI combination.

Similar rates of adverse events in the NRTI group as compared with the other groups and the low incidence of specific NRTI-attributable toxic effects (e.g., renal failure or severe anemia) are reassuring, especially given the challenges of routine laboratory safety monitoring in these settings. When we began this study, the risk-benefit ratio of retaining NRTIs in second-line therapy was unclear, but our findings of substantial efficacy and minimal additional toxicity strongly support their use.

In conclusion, we found that boosted proteaseinhibitor therapy plus two NRTIs, administered without testing of genotypic resistance or regular viral-load monitoring and with limited laboratory safety monitoring, had activity that was not surpassed by alternative regimens considered to be feasible in this setting.

Supported by a grant from the European and Developing Countries Clinical Trials Partnership (IP.2007.33011.003), with funding from the Medical Research Council, United Kingdom; Instituto de Salud Carlos III, Spain (A107/90015); Irish Aid, Ireland; Swedish International Development Cooperation Agency, Sweden; Instituto Superiore di Sanità, Italy; Merck; and the World Health Organization. Substantive in-kind contributions were made by the Medical Research Council Clinical Trials Unit, United Kingdom; CINECA, Bologna, Italy; Janssen Diagnostics, Beerse, Belgium; GlaxoSmithKline/ViiV Healthcare, United Kingdom; and Abbott Laboratories, United States. Trial medication was donated by AbbVie, Merck, Pfizer, GSK, and Gilead. The Malawi-Liverpool-Wellcome Trust Clinical Research Programme, University of Malawi College of Medicine, receives core funding from the Wellcome Trust United Kingdom.

Disclosure forms provided by the authors are available with the full text of this article at NEJM.org.

We thank all the patients and staff from all the centers who participated in this study.

\section{REFERENCES}

1. Gilks CF, Crowley S, Ekpini R, et al. The WHO public-health approach to antiretroviral treatment against HIV in resourcelimited settings. Lancet 2006;368:505-10.

2. Global update on HIV treatment 2013: results, impact and opportunities. Geneva: World Health Organization, 2013.

3. Consolidated guidelines on the use of antiretroviral drugs for treating and preventing HIV infection: recommendations for a public health approach. Geneva: World Health Organization, 2013.

4. Manasa J, Lessells RJ, Skingsley A, et al. High-levels of acquired drug resistance in adult patients failing first-line antiretroviral therapy in a rural HIV treatment programme in KwaZulu-Natal, South Africa. PLoS One 2013;8(8):e72152.

5. Humphreys EH, Chang LW, Harris J. Antiretroviral regimens for patients with HIV who fail first-line antiretroviral therapy. Cochrane Database Syst Rev 2010;6: CD006517.

6. Arribas JR, Delgado R, Arranz A, et al. Lopinavir-ritonavir monotherapy versus lopinavir-ritonavir and 2 nucleosides for maintenance therapy of HIV: 96-week analysis. J Acquir Immune Defic Syndr 2009;51: 147-52.

7. Clumeck N, Rieger A, Banhegyi D, et al. 96 Week results from the MONET trial: a randomized comparison of darunavir/ ritonavir with versus without nucleoside analogues, for patients with HIV RNA $<50$ copies/mL at baseline. J Antimicrob Chemother 2011;66:1878-85.

8. Valantin MA, Lambert-Niclot S, Flandre $\mathrm{P}$, et al. Long-term efficacy of darunavir/ ritonavir monotherapy in patients with HIV-1 viral suppression: week 96 results from the MONOI ANRS 136 study. J Antimicrob Chemother 2012;67:691-5.

9. Antiretroviral therapy for HIV infection in adults and adolescents: recommendations for a public health approach. 2010 revision. Geneva: World Health Organization, 2010.

10. Stanford University HIV drug resistance database (http://hivdb.stanford.edu).

11. WHO case definitions of HIV for surveillance and revised clinical staging and immunological classification of HIV-related disease in adults and children. Geneva: World Health Organization, 2006.

12. Lifson AR, Belloso WH, Davey RT, et al. Development of diagnostic criteria for serious non-AIDS events in HIV clinical trials. HIV Clin Trials 2010;11:205-19.

13. National Institutes of Health, Division of AIDS. Division of AIDS table for grading the severity of adverse events (clarification 9). Bethesda, MD: National Institute of Allergy and Infectious Disease, 2004.

14. Peto R, Pike MC, Armitage P, et al. Design and analysis of randomized clinical trials requiring prolonged observation of each patient. I. Introduction and design. Br J Cancer 1976;34:585-612.

15. Department of Health and Human Services, Food and Drug Administration, Center for Drug Evaluation and Research. Guidance for industry - human immunodeficiency virus-1 infection: developing antiretroviral drugs for treatment. 2013 (http://www.fda.gov/downloads/Drugs/ GuidanceComplianceRegulatoryInformation/ Guidances/UCM355128.pdf). 
16. Idem. Guidance for industry - noninferiority clinical trials. 2010 (http://www .fda.gov/downloads/Drugs/.../Guidances/ UCM202140.pdf).

17. van Buuren S. Multiple imputation of discrete and continuous data by fully conditional specification. Stat Methods Med Res 2007;16:219-42.

18. van Buuren S, Boshuizen HC, Knook DL. Multiple imputation of missing blood pressure covariates in survival analysis. Stat Med 1999;18:681-94.

19. Ajose O, Mookerjee S, Mills EJ, Boulle A, Ford N. Treatment outcomes of patients on second-line antiretroviral therapy in resource-limited settings: a systematic review and meta-analysis. AIDS 2012;26: 929-38.

20. Casotti JA, Passos LN, Oliveira FJ, Ce rutti $\mathrm{C}$ Jr. Factors associated with paradoxical immune response to antiretroviral therapy in HIV infected patients: a case control study. BMC Infect Dis 2011;11:306 21. Zoufaly A, an der Heiden M, Kollan C, et al. Clinical outcome of HIV-infected patients with discordant virological and immunological response to antiretroviral therapy. J Infect Dis 2011;203:364-71.

22. Reynes J, Trinh R, Pulido F, et al. Lopinavir/ritonavir combined with raltegravir or tenofovir/emtricitabine in antiretroviral-naive subjects: 96-week results of the PROGRESS study. AIDS Res Hum Retroviruses 2013;29:256-65.
23. SECOND-LINE Study Group. Ritonavirboosted lopinavir plus nucleoside or nucleotide reverse transcriptase inhibitors versus ritonavir-boosted lopinavir plus raltegravir for treatment of HIV-1 infection in adults with virological failure of a standard first-line ART regimen (SECOND-LINE): a randomised, open-label, non-inferiority study. Lancet 2013;381:2091-9.

24. Negredo E, Massanella M, Puertas MC, et al. Early but limited effects of raltegravir intensification on CD4 $\mathrm{T}$ cell reconstitution in HIV-infected patients with an immunodiscordant response to antiretroviral therapy. J Antimicrob Chemother 2013;68:2358-62.

25. Cooper A, García M, Petrovas C, Yamamoto T, Koup RA, Nabel GJ. HIV-1 causes CD4 cell death through DNAdependent protein kinase during viral integration. Nature 2013;498:376-9.

26. Bartlett JA, Ribaudo HJ, Wallis CL, et al. Lopinavir/ritonavir monotherapy after virologic failure of first-line antiretroviral therapy in resource-limited settings. AIDS 2012;26:1345-54

27. Manosuthi W, Kiertiburanakul S, Amornnimit $\mathrm{W}$, et al. Treatment outcomes and plasma level of ritonavir-boosted lopinavir monotherapy among HIV-infected patients who had NRTI and NNRTI failure. AIDS Res Ther 2009;6:30.

28. Gilks CF, Walker AS, Dunn DT, et al. Lopinavir/ritonavir monotherapy after
24 weeks of second-line antiretroviral therapy in Africa: a randomized controlled trial (SARA). Antivir Ther 2012;17:1363-73. 29. Bunupuradah $T$, Chetchotisakd $P$, Ananworanich J, et al. A randomized comparison of second-line lopinavir/ritonavir monotherapy versus tenofovir/lamivudine/ lopinavir/ritonavir in patients failing NNRTI regimens: the HIV STAR study. Antivir Ther 2012;17:1351-61. [Erratum, Antivir Ther 2012;17:1389-90.]

30. Cameron DW, da Silva BA, Arribas JR, et al. A 96-week comparison of lopinavirritonavir combination therapy followed by lopinavir-ritonavir monotherapy versus efavirenz combination therapy. J Infect Dis 2008;198:234-40.

31. Delfraissy JF, Flandre P, Delaugerre C, et al. Lopinavir/ritonavir monotherapy or plus zidovudine and lamivudine in antiretroviral-naive HIV-infected patients. AIDS 2008;22:385-93.

32. Deeks SG, Wrin T, Liegler T, et al. Virologic and immunologic consequences of discontinuing combination antiretroviraldrug therapy in HIV-infected patients with detectable viremia. N Engl J Med 2001;344: 472-80.

33. Deeks SG, Hoh R, Neilands TB, et al. Interruption of treatment with individual therapeutic drug classes in adults with multidrug-resistant HIV-1 infection. J Infect Dis 2005;192:1537-44.

Copyright (๑) 2014 Massachusetts Medical Society.

\section{JOURNAL ARCHIVE AT NEJM.ORG}

Every article published by the Journal is now available at NEJM.org, beginning with the first article published in January 1812. The entire archive is fully searchable, and browsing of titles and tables of contents is easy and available to all.

Individual subscribers are entitled to free 24-hour access to 50 archive articles per year. Access to content in the archive is available on a per-article basis and is also being provided through many institutional subscriptions. 